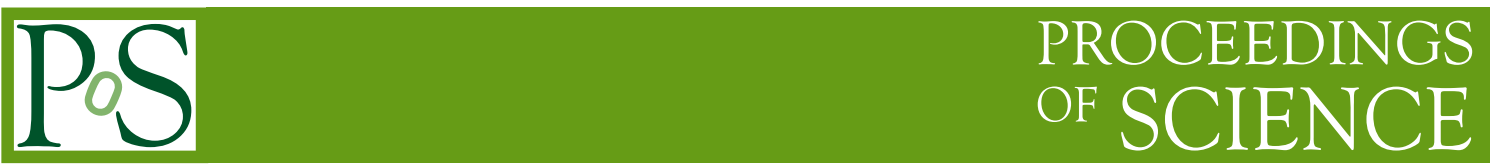

\title{
Yang-Mills Theory at Non-Vanishing Temperature
}

\author{
Leonard Fister* \\ Institut für Theoretische Physik, Universität Heidelberg, Philosophenweg 16, 69120 Heidelberg, \\ Germany \\ E-mail: 1.fisterethphys.uni-heidelberg.de
}

\section{Jan Martin Pawlowski}

Institut für Theoretische Physik, Universität Heidelberg, Philosophenweg 16, 69120 Heidelberg, Germany

E-mail: j.pawlowski@thphys.uni-heidelberg.de

\begin{abstract}
We compute ghost and gluon propagators of Yang-Mills theory in the Landau gauge at nonvanishing temperature within a functional renormalisation group setting. We construct purely thermal flows, that project onto thermal fluctuations only. For temperatures and momenta above the confinement-deconfinement temperature $T_{c}$ the electric propagator shows a thermal suppression due to Debye screening. The magnetic gluon propagator shows a thermal scaling and tends towards the three-dimensional one. In this region both propagators match the lattice propagators. The thermal scaling is also reflected in the infrared suppression of the ghost-gluon vertex. For temperatures below $T_{c}$ the electric propagator shows an enhancement which is in qualitative agreement with the lattice behaviour.
\end{abstract}

International Workshop on QCD Green's Functions, Confinement and Phenomenology, September 05-09, 2011

Trento Italy

\footnotetext{
*Speaker.
} 


\section{Introduction}

In the last decades the phase diagram of quantum chromodynamics (QCD) has been a very active field of research. For small quark chemical potential, but non-vanishing temperature enormous progress has been achieved, which is mainly due to the fact that both, first principle continuum methods, see e.g. $[1,2,3,4,5]$, as well as lattice QCD, see e.g. [6, 7, 8], have been applied very successfully. Nonetheless, even at vanishing density, some questions still remain open, in particular in the vicinity of the chiral and confinement-deconfinement phase transitions. This concerns for example the trace anomaly in this region. Indeed, for temperatures around the deconfinementconfinement phase transition $T \lesssim 3 T_{c}$ continuum methods so far lack quantitative precision. This temperature regime is governed by fully non-perturbative physics, and requires the application of non-perturbative techniques.

In continuum approaches to QCD, it is mainly the glue sector which requires the most effort, in particular conceptually but also technically. In the past two decades much progress has been made in order to understand Yang-Mills theory in the Landau gauge at vanishing temperature, leading to quantitative precision for correlation functions, see [9]. At finite temperature, however, apart from conceptual issues, the same quantitative precision is lacking.

In the present contribution we report on work published in [10]. We aim at a quantitative computation of the Yang-Mills thermal propagators, for a review on thermal gluons see [11]. Here we utilise the functional renormalisation group (FRG), for QCD-related reviews see $[1,12,13$, 14, 15, 16, 17, 18]. The FRG incorporates non-perturbative effects by a successive integration of fluctuations related to a given momentum scale, and hence is applicable to all temperatures. In the presented work we will focus on the pure Yang-Mills part of QCD, which has so far been studied in the framework of functional methods by the help of Dyson-Schwinger equations [11, 19, 20]. We compute fully dressed, non-perturbative ghost and gluon propagators, as well as the ghost-gluon vertex at finite temperature. The electric propagator shows the typical thermal screening above the confinement-deconfinement temperature $T_{c}$ in quantitative agreement with the lattice results, see $[11,20,21,22,23,24,25]$. It also shows an enhancement below $T_{c}$ which is qualitatively similar to the lattice findings. The magnetic propagator shows thermal scaling and tends towards the three-dimensional one for large temperatures. As for the electric propagator, it agrees well with the lattice.

\section{Yang-Mills Green Functions with the Functional Renormalisation Group}

The FRG is an exact method that is derived directly from the functional integral. It constitutes a renormalisation group equation [26] for the scale-dependent effective action $\Gamma_{k}$, which interpolates between the classical action in the ultraviolet and the full effective action in the infrared by variation of an infrared cutoff, which suppresses fluctuations with momenta smaller than the infrared scale $k$. In the limit of $k \rightarrow 0$ one is left with the full quantum effective action.

The classical gauge fixed $S U\left(N_{c}\right)$ Yang-Mills action in Euclidean space in the Landau gauge is given by

$$
S=\int d^{4} x\left(\frac{1}{4} F_{\mu \nu}^{a} F_{\mu \nu}^{a}+\bar{c}^{a} \partial_{\mu} D_{\mu}^{a b} c^{b}\right)
$$




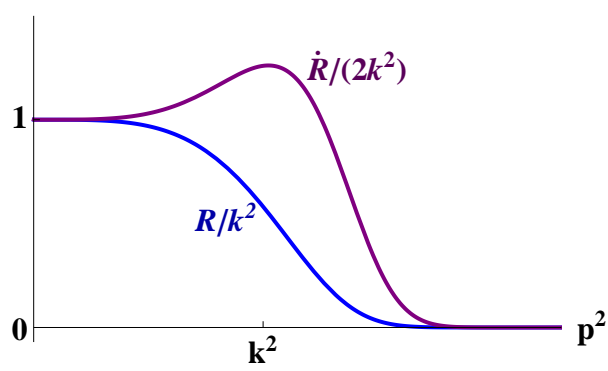

Figure 1: Regulator $R\left(p^{2}\right)$ and its (logarithmic) cut-off scale derivative $\partial_{t} R\left(p^{2}\right)=\dot{R}\left(p^{2}\right)$.

with the ghost fields $\bar{c}^{a}, c^{a}$. The field strength tensor $F_{\mu \nu}^{a}$ and the covariant derivative $D_{\mu}^{a b}$ are defined in terms of the gluons $A_{\mu}^{a}$, the coupling $g$ and the structure constants $f^{a b c}$ of the gauge group,

$$
F_{\mu \nu}^{a}=\partial_{\mu} A_{v}^{a}-\partial_{v} A_{\mu}^{a}+g f^{a b c} A_{\mu}^{b} A_{v}^{c}, \quad D_{\mu}^{a b}=\delta^{a b} \partial_{\mu}+g f^{a c b} A_{\mu}^{c} .
$$

In the FRG-framework, infrared fluctuations below the scale $k$ are suppressed by a modification of the propagator in the classical action of the form $S \rightarrow S+\Delta S_{k}$, with

$$
\Delta S_{k}=\int \frac{d^{4} p}{(2 \pi)^{4}} \frac{1}{2} A_{\mu}^{a} R_{\mu v}^{a b}\left(p^{2}\right) A_{v}^{b}+\int \frac{d^{4} p}{(2 \pi)^{4}} \bar{c}^{a} R^{a b}\left(p^{2}\right) c^{b} .
$$

The regulator functions $R_{\mu \nu}^{a b}$ and $R^{a b}$ carry the same Lorentz and colour structure as the corresponding field propagators and are proportional to the dimensionless shape function $r$ such that schematically $R\left(p^{2}, k^{2}\right) \sim p^{2} r\left(p^{2} / k^{2}\right)$. Note that the ghost regulator is negative related to the unphysical dispersion of the ghost. In the following we use regulators of the form given in Fig. 1. The flow equation for the scale-dependent Yang-Mills effective action $\Gamma_{k}$ at finite temperature is given by

$$
\partial_{t} \Gamma_{k}[\varphi]=\frac{1}{2} \operatorname{Tr} G[\varphi](p, p) \cdot \partial_{t} R_{k}(p) .
$$

The trace stands for summation over species of fields with a relative minus sign for fermions, a summation over Lorentz and colour indices, and the integration/summation of the loop momenta. We also introduced the compact notation $\varphi=(A, c, \bar{c})$. In eq. (2.4) $G[\varphi]$ is the short notation for the fully dressed field dependent propagator. At imaginary time in the Matsubara formalism the integration measure at finite temperature turns the integration over the temporal momentum component into a sum over Matsubara frequencies,

$$
\int \frac{d^{4} p}{(2 \pi)^{4}} \rightarrow T \sum_{n \in \mathbb{Z}} \int \frac{d^{3} p}{(2 \pi)^{3}}, \quad \text { with } \quad p_{0}=2 \pi T n .
$$

The functional flow equation for the effective action (2.4) can be illustrated in a diagrammatic form, which is given in Fig. 2. The minus sign in front of the ghost loops originates in the fermionic nature of the ghost. The flow equations for the propagators can be obtained by taking functional derivatives with respect to two gluons for the gluon propagator, and with respect to a ghost and an antighost for the ghost-propagator. These equations for the Yang-Mills two-point functions are depicted in Fig. 3. They only contain one-loop terms in full propagators and vertices. We emphasise that the flow equation depicted in Fig. 3 is exact, no higher loop terms are missing. This originates in the one-loop form of (2.4), see also Fig. 2. 


\section{Thermal Fluctuations}

The integration of the flow equation (2.4) involves both, quantum as well as thermal fluctuations. However, the treatment of the thermal fluctuations can be done separately via the help of purely thermal flows, which are constructed as the difference of the flows at finite and zero temperature, $[13,27]$,

$$
\partial_{t} \Delta \Gamma_{T, k}[\phi]=\left.\frac{1}{2} \operatorname{Tr} G[\phi] \cdot \partial_{t} R\right|_{T}-\left.\frac{1}{2} \operatorname{Tr} G[\phi] \cdot \partial_{t} R\right|_{T=0} .
$$

The quantity $\Delta \Gamma_{T, k}[\phi]=\left.\Gamma_{k}[\phi]\right|_{T}-\left.\Gamma_{k}[\phi]\right|_{T=0}$ accounts for the difference between the effective action at finite and zero temperature. For the implementation of this procedure the vacuum physics is used as the starting point. A given set of correlation functions at vanishing temperature and scale $k=0$ is integrated by the help of the flow (2.4) up to a scale $\Lambda$, which is chosen such, that all thermal fluctuations are suppressed for the considered temperature $T$. Thus at this scale the correlation functions are not altered at leading order by switching on the temperature. With the flow at finite temperature we can flow down to $k=0$ again, this time integrating thermal fluctuations as well. Note that within this procedure only the difference $\partial_{t} \Delta \Gamma_{T, k}$, i.e. only thermal effects, are sensitive to the chosen approximation.

The idea of the thermal flow is illustrated in a heuristic plot in Fig. 4. However, note that for increasing renormalisation group scale $k$ the decay $\Delta \Gamma_{T, k}^{(n)}\left(p_{1}, \ldots, p_{n}\right) \rightarrow 0$ is exponential with exponent $k / T$ but only polynomially in $p_{n}^{2} / k^{2}$, which is an important issue for numerical accuracy of thermodynamic quantities. This polynomial suppression is due to the fact, that only the loopmomenta are constrained by the regulator $q \lesssim k$, but the external momenta $p$ are not limited. For large momenta $p \gg k$ the flow factorises at leading order, see Fig. 5, which weakens the locality of the flow. However, the locality of a flow can be restored by a $k$-dependent reparametrisation of the fields, which can be done in such a way, that the flow of the two-point functions is strictly zero for external momenta larger than a certain scale $p>\lambda k,[15,10]$. Note that the contributions stemming from this region are not dropped, but rather stored in a reparametrisation function of the fields. This reparametrisation must be undone at the end of the computation. In the following we will drop any reference to the reparametrisation of fields and refer the interested reader to [10].

\section{Approximation}

The flow of an arbitrary $n$-point function depends itself on $m$-point functions with $m \leq n+2$, see e.g. that of the propagators depicted in Fig. 3. As a consequence, the flow in eq. (2.4) generates an infinite hierarchy of coupled integro-differential equations. Within numerical applications

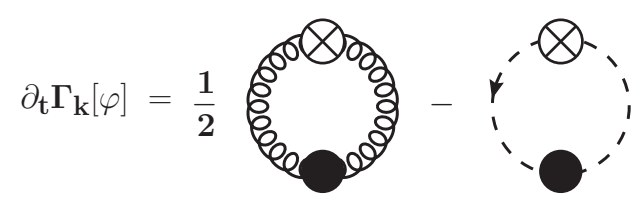

Figure 2: Functional flow for the effective action. Lines with filled circles denote fully dressed field dependent propagators $G[\varphi]$. Crossed circles denote the regulator insertion $\partial_{t} R_{k}$. 


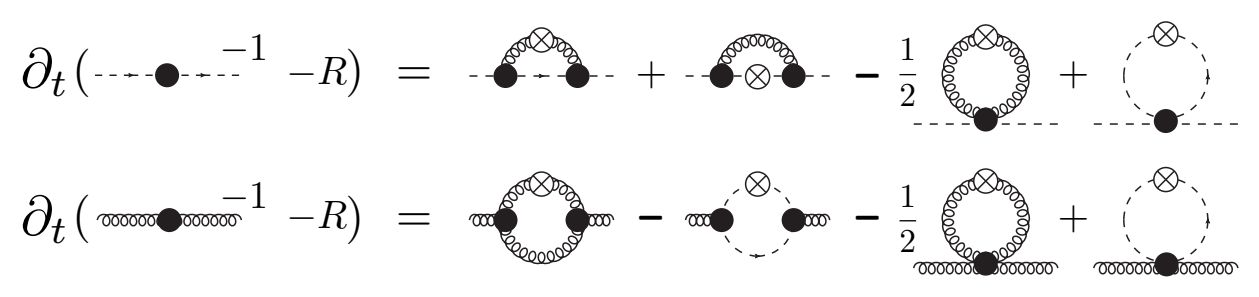

Figure 3: Flow equations for the ghost and gluon propagators. Vertices with filled circles denote fully dressed vertices. All propagators are fully dressed, the filled circles for the internal ones have been dropped for the sake of clarity. Crossed circles denote the regulator insertion $\partial_{t} R_{k}$.

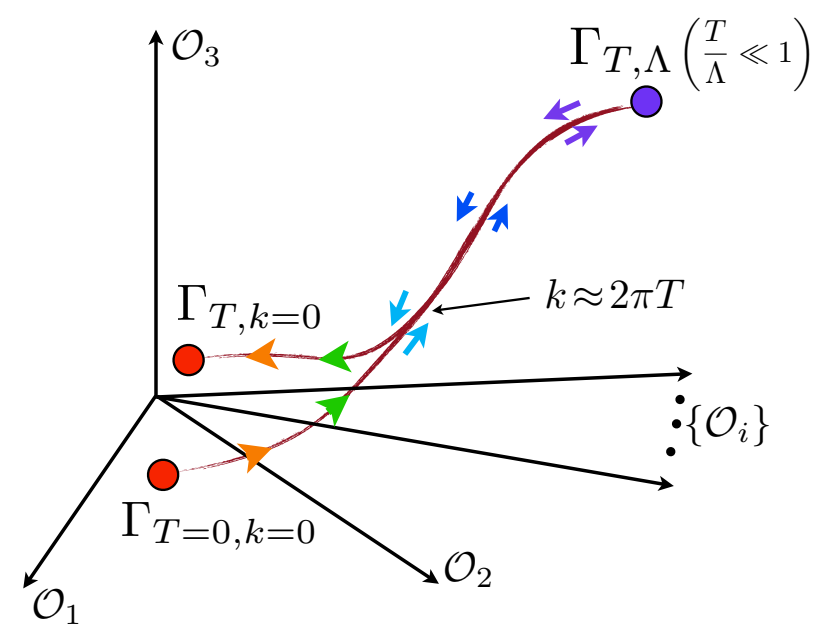

Figure 4: Flow at vanishing temperature from $k=0$ to $k=\Lambda$, and flow at $T \neq 0$ from $k=\Lambda$ to $k=0$ with $T / \Lambda \ll 1$. The flow is described in theory space, and the axes label (orthogonal) couplings/observables $\mathscr{O}_{i}$ which serve as expansion coefficients of the effective action, e.g. $\mathscr{O}_{1}=\Gamma^{(2)}(p=0)$. The flows start to deviate at $k \approx 2 \pi T$.

this system must be closed by projecting it on a finite set of flow equations while keeping the relevant physics. In other words, the contributing correlation functions must already comprise the relevant physics. For the propagators we keep the full propagators and work with self-consistent approximations to the vertices which respect the renormalisation group properties of the vertices. Furthermore, we use the flow equation for the ghost-gluon vertex at the symmetric point with $p^{2}=k^{2}$.

In the Landau gauge the gluon is a purely transversal vector particle. At finite temperature the heat bath singles out a preferred rest frame defined by a time-like vector $n_{\mu}$. This leaves us with one $(3 d)$ longitudinal and one transversal tensor structure, both being transverse in $4 d$. The 


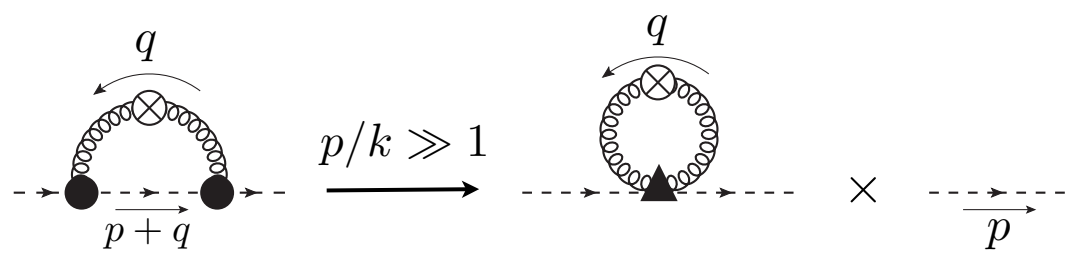

Figure 5: Factorisation in leading order for large momenta for the first diagram (cutted gluon line) in the flow of the (inverse) ghost propagator given in Fig. 3. The triangle stands for the product of the two vertices at $q=0$ and reads $-p_{\mu} p_{\mu^{\prime}} f^{a c d} f^{b c^{\prime} d^{\prime}}$.

corresponding projection operators are given by

$$
\begin{aligned}
\Pi_{\mu \nu}^{T}(p) & =\delta_{\mu \nu}-p_{\mu} p_{v} / p^{2}, \\
P_{\mu \nu}^{T}\left(p_{0}, \vec{p}\right) & =\left(1-\delta_{\mu 0}\right)\left(1-\delta_{v 0}\right)\left(\delta_{\mu \nu}-p_{\mu} p_{\nu} / \vec{p}^{2}\right), \\
P_{\mu \nu}^{L}\left(p_{0}, \vec{p}\right) & =\Pi_{\mu \nu}^{T}(p)-P_{\mu \nu}^{T}\left(p_{0}, \vec{p}\right) .
\end{aligned}
$$

At vanishing temperature the gluon can be parametrised with one scalar function. At non-vanishing temperature the wave-function renormalisations for the longitudinal propagator $Z_{L}$ and transverse propagator $Z_{T}$ differ and have to be taken into account separately. The ghost has no Lorenz structure and can be described fully in terms of one scalar function also at non-vanishing temperature. Leaving out the identity in colour-space the parametrisation is done according to

$$
\begin{aligned}
\left(\Gamma_{A, L}^{(2)}\right)_{\mu \nu}\left(p_{0}, \vec{p}\right) & =Z_{L}\left(p_{0}, \vec{p}\right) p^{2} P_{\mu \nu}^{L}\left(p_{0}, \vec{p}\right), \\
\left(\Gamma_{A, T}^{(2)}\right)_{\mu \nu}\left(p_{0}, \vec{p}\right) & =Z_{T}\left(p_{0}, \vec{p}\right) p^{2} P_{\mu \nu}^{T}\left(p_{0}, \vec{p}\right), \\
\Gamma_{c}^{(2)}\left(p_{0}, \vec{p}\right) & =Z_{c}\left(p_{0}, \vec{p}\right) p^{2},
\end{aligned}
$$

where we emphasise that the wave-function renormalisations depend on the temporal momentum component $p_{0}$ and the spatial ones $\vec{p}$ separately.

The flow equations for the two-point functions depend on the two-, three- and four-point functions. In particular we have tadpole diagrams which depend on the ghost-ghost and ghost-gluon scattering vertices $\Gamma_{\bar{c} c \bar{c} c}^{(4)}$ and $\Gamma_{\bar{c} A^{2} c}^{(4)}$. Albeit these vertices are absent on the classical level they become relevant in the non-perturbative regime. They can be taken into account via a DSE resummation of the vertices in the flow. This turns the flow into the total scale derivative of the DSE, see Fig. 7(a). This reflects the fact that, in general, the flow equation is the differential form of the Dyson-Schwinger equation. We remark that this usually would introduce the necessity of renormalising the corresponding DSE. In the present parameterisation this is avoided due to locality, for details see [10]. As a result the total scale derivative of the DSE for the ghost two-point function in the presence of a regulator term is equivalent to the flow equation, but is free of four-point functions, for details see [10]. The scale derivative acting on the dressed propagator gives

$$
\partial_{t} G[\phi]=-G[\phi] \cdot \partial_{t}\left(\Gamma^{(2)}[\phi]+R_{\phi}\right) \cdot G[\phi] .
$$




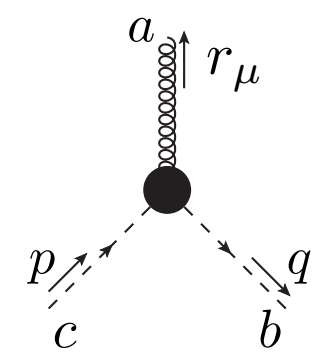

Figure 6: Ghost-gluon vertex.

For the vertices we introduce a parametrisation that naturally captures the renormalisation group behaviour, for details see [28]. With the wave-function renormalisation of the ghost and gluon we write

$$
\Gamma^{(n)}\left(p_{1}, \ldots, p_{n}\right)=\prod_{i=1}^{n} Z_{\phi_{i}}^{1 / 2}\left(p_{i}\right) \mathscr{T}\left(p_{1}, \ldots, p_{n}\right) .
$$

The $Z_{\phi}(p)$-factors carry the RG-scaling of the vertex as well as the momentum dependence, thus they take into account potential kinematic singularities. The $\mathscr{T}$ are renormalisation group invariant tensors, which carry the canonical momentum dimension as well as the tensor and colour structure. For the ghost propagator we have sketched the idea how the ghost-ghost and ghost-gluon scattering vertices can be absorbed via a DSE resummation. With a similar resummation in the flow of the ghost-gluon vertex in Fig. 6 we can fully resolve its RG running at the symmetric point $p^{2}=k^{2}$. We use the parametrisation

$$
\mathscr{T}_{\bar{c} A c, \mu}^{a b c}(q, p)=z_{k, \bar{c} A c} \frac{1}{g}\left[S_{\bar{c} A c}^{(3)}(q, p)\right]_{\mu}^{a b c}=z_{k, \bar{c} A c} i q_{\mu} f^{a b c},
$$

where $g$ is the classical coupling. The RG invariant factor $z_{k, \bar{c} A c}$ defines the running coupling $\bar{\alpha}_{s}=z_{k, \bar{c} A c}^{2} /(4 \pi)$. Also for the three- and four-gluon vertices we restrict ourselves to the classical tensor and colour structure. The parametrisation is done according to eq. (4.4), with

$$
\mathscr{T}_{A^{3}}=z_{k, A^{3}} \frac{1}{g} S_{A^{3}}^{(3)}, \quad \mathscr{T}_{A^{4}}=z_{k, A^{4}} \frac{1}{g^{2}} S_{A^{4}}^{(4)} .
$$

For large momenta the couplings $z_{k, A^{3}}, z_{k, A^{4}}$ relate to the ghost-gluon dressing $z_{k, \bar{c} A c}$. In the infrared the couplings are suppressed strongly. This is taken into account via functions that approach $z_{3}=z_{4}=1$ for $k \gg \Lambda_{Q C D}$, but suppress the gluonic vertices in the infrared. The couplings are given by $z_{k, A^{3}}=z_{3} z_{k, \bar{c} A c}, z_{k, A^{4}}=z_{4} z_{k, \bar{c} A c}^{2}$. In addition to that we regularise the vertex prefactors in such a way that we freeze the $Z_{A}$ for scales smaller than the minimal turning point in the wave-function renormalisation. The naive ansatz of the pure wave-function renormalisation would yield unphysical IR divergent flows, which would spoil the IR suppression of the purely gluonic vertices. The above approximation scheme is depicted in Fig. 7, including the DSE resummations.

\section{Results for Propagators and the Ghost-Gluon Vertex}

In this section we present the results for the ghost and gluon propagators and the ghost-gluon vertex. The temperature is given in lattice units. Only below the typical temperature scale of $2 \pi T$ 


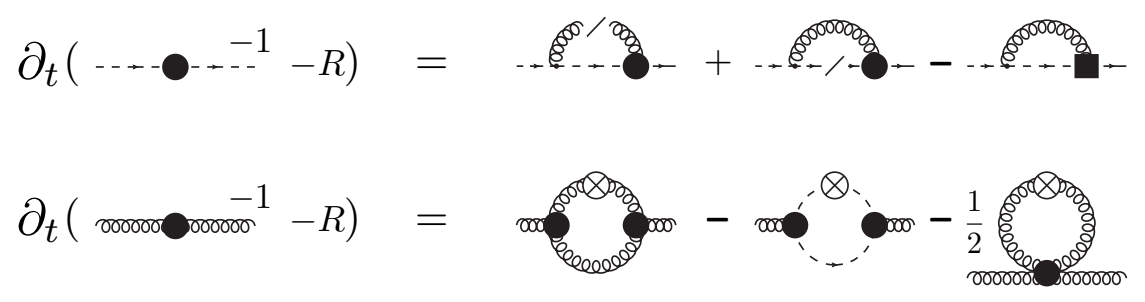

(a) Flows for the two-point functions in the given truncation.

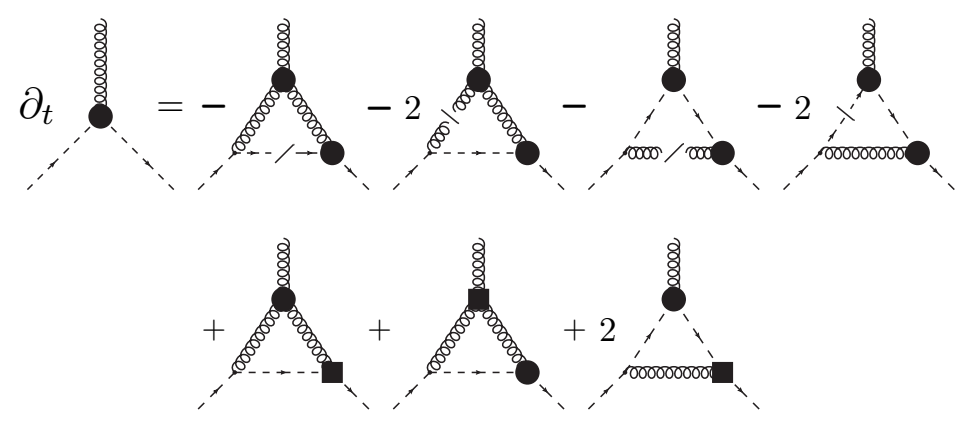

(b) Flow for the ghost-gluon vertex in the given truncation.

Figure 7: Yang-Mills flows for propagators (a) and ghost-gluon vertex (b) the approximation discussed above. The flows for the ghost propagator and the ghost-gluon vertex are DSE-resummed and the ghosttadpole in the gluon equation is neglected.

we have significant thermal effects on the momentum dependence. In turn, above this scale the temperature fluctuations are suppressed and all propagators tend towards their vacuum counterparts at vanishing temperature. This also holds true for the ghost-gluon vertex.

The most significant effect can be seen for the chromoelectric and chromomagnetic gluon propagators, the components of the propagator longitudinal and transversal to the heat bath respectively. The zero mode of the longitudinal gluon propagator at various temperatures is given in Fig. 8(a) as a function of spatial momentum.

For low temperatures $T \lesssim 150 \mathrm{MeV}$ we see an enhancement of the longitudinal propagator. Such an enhancement is also seen on the lattice, [11, 20, 21, 22, 23, 24, 25]. For higher temperatures the longitudinal propagator is suppressed relative to the gluon propagator at vanishing temperature. This is the expected behaviour caused by the Debye screening mass due to the thermal screening of the chromoelectric gluon. For asymptotically high temperatures $\gg T_{c}$ the chromoelectric gluon decouples. The onset of this behaviour at about $T \approx 150 \mathrm{MeV}$ is earlier as in the respective lattice computations $[11,20,21,22,23,24,25]$, where the thermal decoupling takes place for temperatures larger than the critical temperature.

In order to quantitatively capture this behaviour we have to extend our present truncation with a self-consistent inclusion of the Polyakov loop background as well as a better resolution of the purely gluonic vertices for momenta and frequencies below $\Lambda_{\mathrm{QCD}} \propto T_{c}$. Above the confinementdeconfinement scale we see quantitative agreement with the lattice results.

The transversal mode is not enhanced for small temperatures in clear distinction to the longi- 


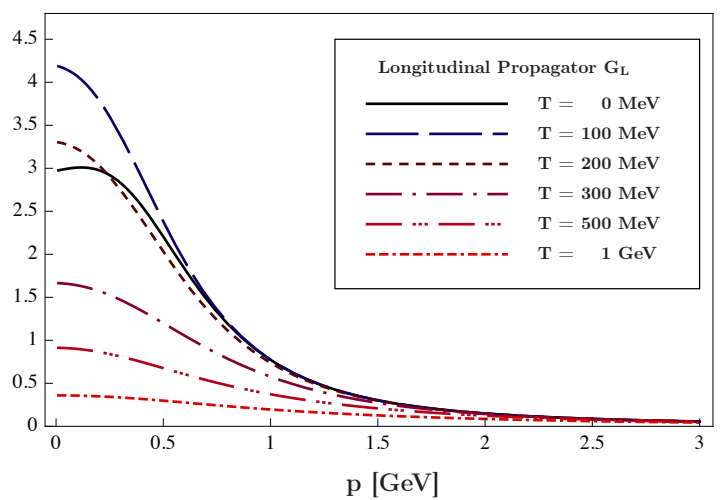

(a) Zero mode of the longitudinal propagator.

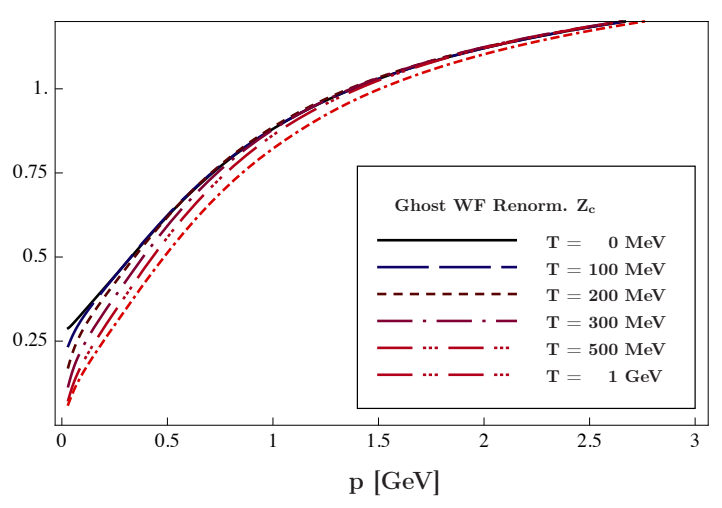

(c) Zero mode of the ghost wave function renormalisation.

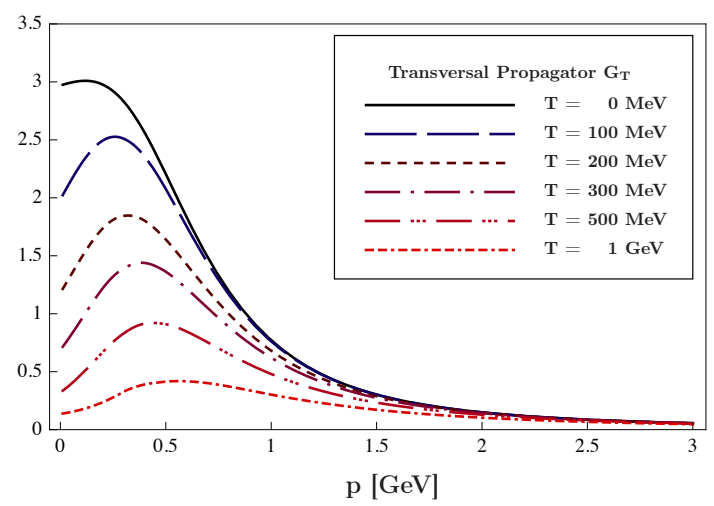

(b) Zero mode of the transverse propagator.

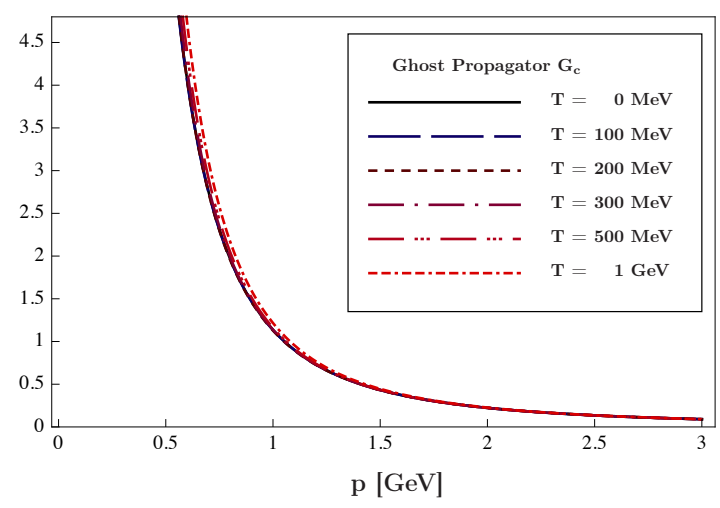

(d) Zero mode of the ghost propagator.

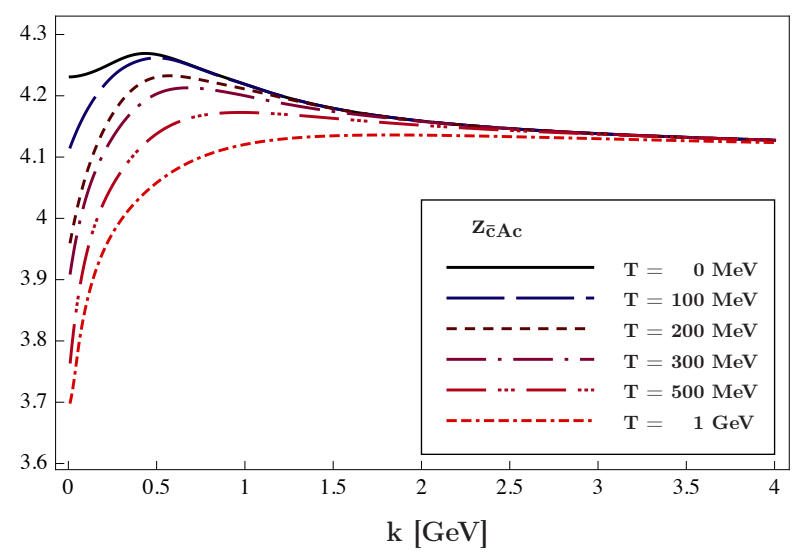

(e) Ghost-gluon vertex coupling.

Figure 8: Yang-Mills propagators and the ghost-gluon vertex at various temperatures.

tudinal mode. It is monotonously decreased with temperature, see Fig. 8(a). Moreover, it develops a clear peak at about $500 \mathrm{MeV}$. This can be linked to positivity violation which has to be present for the transversal mode as in the high temperature limit it describes the remaining dynamical glu- 
ons of three-dimensional Yang-Mills theory in the Landau gauge. The infrared bending is more pronounced as that of respective lattice results, which already holds at vanishing temperature. It is another choice for the decoupling solution as discussed in [9]. Moreover, its strength may also depend on the quantitative precision achieved for the gluonic vertices at these scales. In turn, for larger momenta the transversal propagator agrees well with the respective lattice propagator.

The ghost shows only a small temperature dependence in contradistinction to the gluonic propagators. The temperature dependence is hardly evident on the level of the propagator Fig. 8(d), but can be resolved on the level of the wave-function renormalisation Fig. 8(c). The wave-function renormalisation is slightly suppressed, which corresponds to a successive enhancement of the ghost propagator at finite temperature. The enhancement of the ghost propagator is potentially self-amplifying as it feeds back into the flow of the ghost two-point function, see Fig. 7. This would cause a pole in the ghost propagator at some temperature if the ghost-gluon vertex would not change. However, in this case the flow of the latter is dominated by the ghost diagram, see Fig. 6. This non-trivial interplay of the flow of the ghost propagator with the flow of the ghostgluon vertex leads to a self-stabilising system and prevents a further enhancement of the ghost. This effect is crucial for the stability of the solution of the Yang-Mills system at finite temperature. Indeed, for a constant vertex no solution could be obtained for intermediate temperatures $T \approx T_{c}$ and above. Thus we conclude that any reliable truncation must comprise direct thermal effects also in $n$-point functions with $n \leq 3$.

The self-stabilising property of the Yang-Mills system explained above is clearly seen in the temperature-dependence of the ghost-gluon vertex. The vertex is suppressed successively with the temperature, see Fig. 8(e), which in turn ensures the relatively mild change of the ghost propagator. Especially the sharp drop-off of the vertex at small scales $k$ accounts for the smallness of the thermal fluctuations to the ghost propagator.

In the following, we compare the propagators above with lattice results [21]. For this purpose, we scale the lattice data such that the lattice propagators at vanishing temperature match our normalisation at momenta $p \gtrsim 1 \mathrm{GeV}$. Take notice, that we did not use the lattice propagator as the initial condition, thus the deep infrared of the data deviates from our propagator already at zero temperature, which persists also in the propagators at finite temperature. Apart from that, there is quantitative agreement with the lattice data with respect to the (temperature dependent) momentum region, where the thermal effects appear. In Fig. 9(b) the transversal propagators are compared.

Clearly, we match the lattice propagator, except for the strong bending of the FRG propagator in the infrared region. As already discussed above, this difference is a direct consequence of the deviation of ghost and gluon propagators at vanishing temperature in the deep infrared, and the ansatz for the gluonic vertices. However, the behaviour of the magnetic gluon agrees with the lattice observations for momenta above $500 \mathrm{MeV}$ and all temperatures. In contrast to this, the electric gluon, see Fig. 9(a) on the lattice shows a qualitatively different behaviour for temperatures below and around the phase transition. Note also that although the longitudinal propagators agree for $T=0.361 T_{c} \approx 100 \mathrm{MeV}$, it is exactly this region where the uncertainty due to the truncation for the gluonic vertices is potentially large, for a detailed discussion see [10]. Being aware of a possible truncation dependence in the deep infrared of the longitudinal propagator at low temperatures, we note that in the present truncation the electric gluon qualitatively shows the enhancement found on the lattice. Increasing the temperature this feature disappears, and we see a qualitatively different 


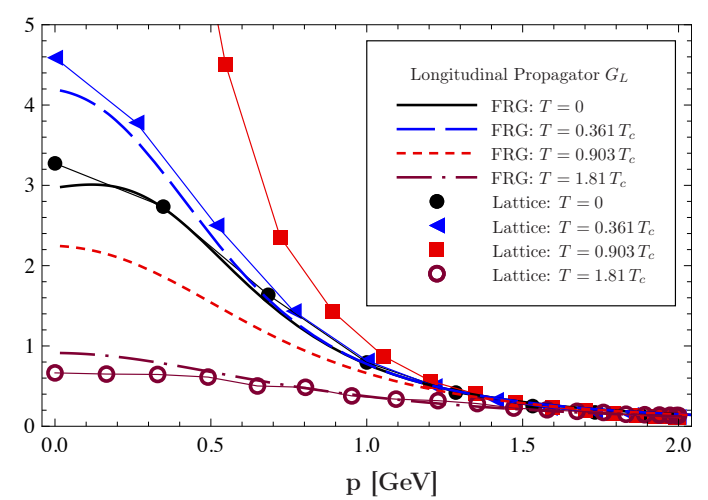

(a) Comparison of the longitudinal propagator from above with the corresponding lattice result.

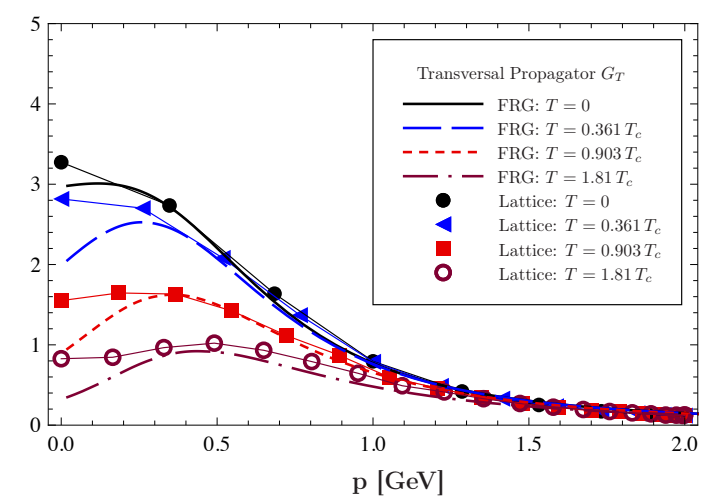

(b) Comparison of the transversal propagator from above with the corresponding lattice result.

Figure 9: Comparison of the gluon propagator with the lattice results

effect for temperatures below $T_{c}$. While the continuum result shows a strictly monotonic decreasing propagator, the counterpart on the lattice is enhanced in the confining regime, but reflects the phase transition in form of a rapid decrease at $T_{c}$. Nevertheless, this deflection is expected to be missed in the present truncation, as the full Polyakov loop potential $V\left(A_{0}\right)$, see $[29,30]$, is pivotal for the critical behaviour around the phase transition. In a full calculation the inverse propagator is proportional to the second derivative of the Polyakov loop $\Gamma_{A, L}^{(2)} \sim V^{\prime \prime}\left(A_{0}\right)$, however in the work presented here this term was dropped. In any case this term is absent in the magnetic modes.

\section{Summary and Outlook}

We have computed temperature dependent Yang-Mills propagators in Landau gauge in the framework of the functional renormalisation group for $T \lesssim 3 T_{c}$. For this purpose we employ thermal flows, which encode thermal fluctuations in the difference of the full renormalisation group flow at non-vanishing and zero temperature.

The chromoelectric propagator shows the expected Debye-screening for $T>T_{c}$ in quantitative agreement with the lattice results. For small temperatures it shows qualitatively the enhancement also seen on the lattice $[11,20,21,22,23,24,25]$. However, the significance of the lattice results so far as well as quantitative details are not settled yet. We hope to add something to the clarification of this issue within an extension of the present work. There we resolve the dependence on the Polyakov loop as well as have a better grip on the gluonic vertices. The chromomagnetic propagator shows the expected thermal scaling and tends towards the three-dimensional gluon propagator in quantitative agreement with the lattice.

The ghost propagator only shows a mild enhancement with temperature in agreement with the lattice. In contradistinction we see a strong thermal infrared suppression of the ghost-gluon vertex which increases with temperature.

At present we improve on the approximations and compute thermodynamic observables, e.g. the pressure. Furthermore, we extend our work to full QCD with 2 and $2+1$ flavours in an extension 
of [31] also at finite density.

\section{References}

[1] J. M. Pawlowski, AIP Conf.Proc. 1343, 75 (2011), 1012.5075.

[2] D. Binosi and J. Papavassiliou, Phys. Rept. 479, 1 (2009), 0909.2536.

[3] C. S. Fischer, J.Phys.G G32, R253 (2006), hep-ph/0605173.

[4] R. Alkofer and L. von Smekal, Phys. Rept. 353, 281 (2001), hep-ph/0007355.

[5] C. D. Roberts and S. M. Schmidt, Prog. Part. Nucl. Phys. 45, S1 (2000), nucl-th/0005064.

[6] O. Philipsen, (2011), 1111.5370.

[7] S. Borsanyi et al., JHEP 1011, 077 (2010), 1007.2580.

[8] HotQCD collaboration, A. Bazavov and P. Petreczky, J.Phys.Conf.Ser. 230, 012014 (2010), 1005.1131 .

[9] C. S. Fischer, A. Maas, and J. M. Pawlowski, Annals Phys. 324, 2408 (2009), 0810.1987.

[10] L. Fister and J. M. Pawlowski, in preparation (2011).

[11] A. Maas, (2011), 1106.3942.

[12] D. F. Litim, (1998), hep-ph/9811272.

[13] D. F. Litim and J. M. Pawlowski, World Sci. , 168 (1999), hep-th/9901063.

[14] J. Berges, N. Tetradis, and C. Wetterich, Phys.Rept. 363, 223 (2002), hep-ph/0005122.

[15] J. M. Pawlowski, Annals Phys. 322, 2831 (2007), hep-th/0512261.

[16] H. Gies, (2006), hep-ph/0611146.

[17] B.-J. Schaefer and J. Wambach, Phys.Part.Nucl. 39, 1025 (2008), hep-ph/0611191.

[18] J. Braun, (2011), 1108.4449.

[19] B. Grüter, R. Alkofer, A. Maas, and J. Wambach, Eur. Phys. J. C42, 109 (2005), hep-ph/0408282.

[20] A. Cucchieri, A. Maas, and T. Mendes, Phys. Rev. D75, 076003 (2007), hep-lat/0702022.

[21] C. S. Fischer, A. Maas, and J. A. Müller, Eur. Phys. J. C68, 165 (2010), 1003.1960.

[22] V. Bornyakov and V. Mitrjushkin, (2011), 1103.0442.

[23] R. Aouane et al., (2011), 1108.1735.

[24] A. Maas, J. M. Pawlowski, L. von Smekal, and D. Spielmann, (2011), 1110.6340.

[25] A. Cucchieri and T. Mendes, (2011), 1105.0176.

[26] C. Wetterich, Phys.Lett. B301, 90 (1993).

[27] D. F. Litim and J. M. Pawlowski, JHEP 0611, 026 (2006), hep-th/0609122.

[28] C. S. Fischer and J. M. Pawlowski, Phys. Rev. D80, 025023 (2009), 0903.2193.

[29] J. Braun, H. Gies, and J. M. Pawlowski, Phys. Lett. B684, 262 (2010), 0708.2413.

[30] J. Braun, A. Eichhorn, H. Gies, and J. M. Pawlowski, Eur.Phys.J. C70, 689 (2010), 1007.2619.

[31] J. Braun, L. M. Haas, F. Marhauser, and J. M. Pawlowski, Phys. Rev. Lett. 106, 022002 (2011), 0908.0008. 